\title{
Orientational Coupling Amplification in Ferroelectric Nematic Colloids
}

\author{
Fenghua $\mathrm{Li}^{1}$, Oleksandr Buchnev ${ }^{2}$, Chae Il Cheon ${ }^{3}$, Anatoliy Glushchenko ${ }^{4}$, \\ Victor Reshetnyak $^{5}$, Yuri Reznikov ${ }^{2}$, Timothy J. Sluckin ${ }^{6}$ and John L. West ${ }^{1}$ \\ ${ }^{1}$ Liquid Crystal Institute, Kent State University, Kent, OH 44242, USA \\ ${ }^{2}$ Institute of Physics, Ukrainian Academy of Science, Kyiv, Ukraine \\ ${ }^{3}$ Hoseo University, Baebang, Asan, Chungnam, 336-795, Korea \\ ${ }^{4}$ University of Colorado at Colorado Springs, Colorado Springs, CO 80933, USA \\ ${ }^{5}$ Kyiv Taras Shevchenko University, Kyiv, Ukraine and \\ ${ }^{6}$ School of Mathematics, University of Southampton, Southampton SO17 1BJ, United Kingdom
}

(Dated: March 15, 2006; Received textdate; Accepted textdate)

\begin{abstract}
We investigated the physical properties of low concentration ferroelectric nematic colloids, using calorimetry, optical methods, infrared spectroscopy and capacitance studies. The resulting colloids normally remain homogeneous, but the nematic orientational coupling is significantly amplified. In particular cases, the nematic orientation coupling increases by $10 \%$ for particle concentrations of $0.2 \%$. A manifestation of the increased orientational order is that the clearing temperature of a nematic colloid increases up to 40 Celsius degrees compared to the pure LC host. A theoretical model is proposed in which the ferroelectric particles induce local dipoles whose effective interaction is proportional to the square of the orientational order parameter.
\end{abstract}

PACS numbers: 64.70.Md, 82.70.Dd, 77.84.-s

Colloids in which the solute is liquid crystalline (LC) are known to possess an extremely rich set of behaviors [1-5]. The anchoring between the LC and microcolloidal particles $(\geqslant 1 \mu \mathrm{m})$ can produce long-range orientational distortions around the particles. This results in strong inter-particle interactions - sometimes repulsive and sometimes attractive - in the mesophase. The interactions can give rise to well-ordered structures of particles in the liquid crystal matrix (both lattices and chains) [2, 3]. However, in most cases a prerequisite for interesting LC colloidal behavior has been a high concentration dispersion, typically with particle volume fraction $\mathrm{c}_{\text {part }} \geqslant 30 \%$. In such systems aggregated particles produce director distortions extending over macroscopic scales. These suspensions scatter light strongly, and possess unique structural, mechanical, electro- and magneto-optical properties [5, 6].

Recently, we have shown that even at low concentrations $\left(\mathrm{c}_{\text {part }} \leqslant 1 \%\right)$, LC colloids differ strongly from the pure host material [7-10]. These colloids consist of submicron ferroelectric particles suspended in the LC host. In these systems, unlike in classic LC colloids, the suspensionmatrix interaction is insufficient to disturb the LC orientation. This small concentration dramatically increases the dielectric anisotropy, significantly decreases the Freedericksz transition voltage, and significantly accelerates electric field-induced director reorientation.

In this letter, we report results which show that these phenomena are general properties of liquid crystal suspensions containing ferroelectric colloidal nanoparticles. The addition of impurities normally decreases the nematic clearing temperature $T_{N I}$ [11]. However, our measurements show massive increases in $T_{N I}$, of the order of $40^{\circ} \mathrm{C}$, for mass impurity concentrations of the order of $0.2 \%$. These results imply an increase in the effective nematic in- teraction parameter. Measurements of the birefringence, dielectric anisotropy and order parameter in the suspension are consistent with this picture.

We have also constructed a theoretical model. The ferroelectric particles in the suspension produce large electric fields in their neighborhood. These electric fields produce induced dipoles on the nematic molecules, and the mean magnitude of the induced dipoles is proportional to the average polarizability and hence the nematic order parameter. The dipolar interaction between the induced dipoles is thus proportional to the nematic order parameter, and provides an additional component to the effective nematic interaction. Order of magnitude estimates of the magnitude of this quantity are consistent with our observations.

Most of our experiments utilized the nematic LC MLC6609 from Merck $\left(T_{N I}=91.5^{\circ} \mathrm{C}\right)$, doped with $\mathrm{BaTiO}_{3}$ nanoparticles from Aldrich. The nanoparticle dimension was 50-100 nm (transmission electron microscopy). The spontaneous polarization of the monodomain particles is assumed the same as that of $\mathrm{BaTiO}_{3}$ monocrystal, 0.26 $\mathrm{C} \mathrm{m}^{-2}$ [12]. The detailed preparation process of the colloid was described recently (see Ref. [7]).

The clearing temperature $T_{N I}$ was determined using DSC at a rate of $5 \mathrm{~K} \mathrm{~min}^{-1}$. The resulting DSC scans for the pure MLC-6609 and for the colloid ( $\mathrm{c}_{\text {part }} \approx 0.2 \%$ ) are shown in Fig. 1. When the $\mathrm{BaTiO}_{3}$ nanoparticles are introduced, the clearing temperature increases by $38.7^{\circ} \mathrm{C}$. A lower mass fraction $\left(\mathrm{c}_{\text {part }} \approx 0.05 \%\right)$ produced a reduced shift $\Delta T_{N I}=23.1^{\circ} \mathrm{C}$. The changes in $T_{N I}$ were confirmed using polarized optical microscope.

We also measured other LC properties in the colloidal suspension, in order to check the hypothesis that the increase in $T_{N I}$ is a consequence solely of an increased effective nematic interaction. Firstly we measured order param- 


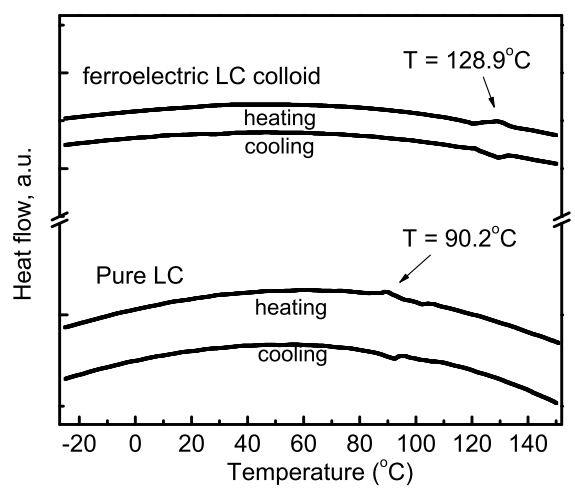

FIG. 1: DSC-graphs for heating and cooling of the samples with pure LC MLC-6609 (bottom pair of the curves) and colloids of BaTiO3 in MLC-6609 (top pair of the curves), $\mathrm{c}_{\text {part }} \approx 0.2 \%$.

eters, using FTIR spectroscopy and observing the dichroism of the characteristic functional groups of the nematic matrix components. We used the stretch vibration of $\mathrm{C}=\mathrm{C}$ groups of benzene rings $\left(\nu=1513 \mathrm{~cm}^{-1}\right)$ because this group is oriented along the long axis of the LC molecules. The $\mathrm{C}=\mathrm{C}$ band dichroism was measured using a Magna 550 FTIR (Nicolet) spectrometer in $\mathrm{NaCl}$ cells of $16 \mu \mathrm{m}$ thickness. The LC and the colloid were homogeneously aligned using a thin $(<0.1 \mu \mathrm{m})$ rubbed polyimide alignment layer. The order parameter was evaluated using the formula $S=(D-1) /(D+2)$, where $D=A_{/ /} / A_{\perp}$ is the dichroism of absorbance parallel $\left(A_{/ /}\right)$, and perpendicular $\left(A_{\perp}\right)$ to the IR beam polarization. Fig. 2 shows the temperature dependence of the resulting order parameters, both in the pure MLC-6609 and in the ferroelectric colloid $\left(\mathrm{c}_{\text {part }} \approx 0.2 \%\right)$. The clearing temperature increase is mirrored by an order parameter increase; at $30^{\circ} \mathrm{C}$ the order parameter gain, $S_{C O L} / S_{L C}=1.2$.

A further measurement compares the optical birefringence of the colloid to that of the pure nematic. We assume the ferroelectric nanoparticles act as effective molecular dopants. The birefringence of the colloid can then be written as [13]:

$$
n_{a}^{C O L}=\frac{4 \pi F N_{L C} \gamma_{a}^{C O L}}{n_{e}^{C O L}+n_{o}^{C O L}} S_{C O L}
$$

where $F$ is the local field factor, $\gamma_{a}^{C O L}=\left(1-c_{\text {part }}\right) \gamma_{a}^{L C}+$ $c_{\text {part }}\left(\gamma_{a}^{\text {part }}\right)$ with $\gamma_{a}^{L C}$ is the LC molecular polarizability anisotropy and $\gamma_{a}^{\text {part }}$ is the polarizability anisotropy of the ferroelectric particles. The birefringence gain with respect to the pure $\mathrm{LC}$ is then given by:

$$
\frac{n_{a}^{C O L}}{n_{a}^{L C}}=\frac{\left(n_{e}^{L C}+n_{o}^{L C}\right)}{\left(n_{e}^{C O L}+n_{o}^{C O L}\right)} \frac{\gamma_{a}^{C O L}}{\gamma_{a}^{L C}} \frac{S_{C O L}}{S_{L C}}
$$

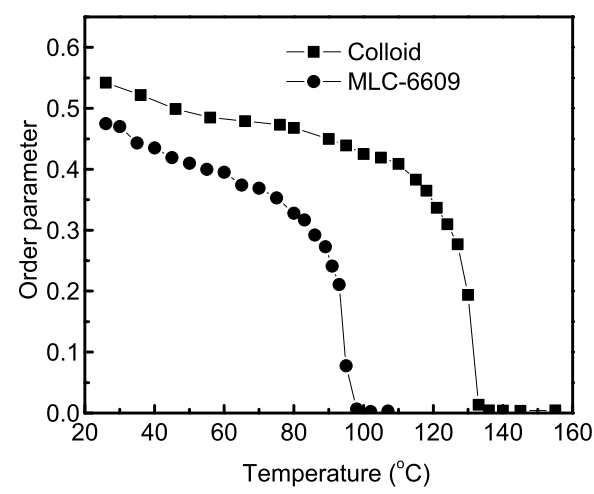

FIG. 2: Temperature dependences of the order parameter of MLC-6609 and the ferroelectric colloid ( $\left.\mathrm{c}_{\text {part }} \approx 0.2 \%\right)$.

The birefringence gain in the colloid involves three separate components. The first is the statistical mechanicsinduced order parameter increase. The second depends also on the effective anisotropy of both the ferroelectric particles and LC molecules, the values of which are not known. Finally, the gain depends on the ratio of the refractive indices of the LC and the colloid. We measured these refractive indices and find that the ratio is essentially unity.

Experimentally we obtained $n_{a}(T)$ using an Abbé refractometer. An independent set of experiments used a retardation technique measuring the phase shift between eand o-waves $\varphi=\pi d n_{a} / \lambda$ in planar cells $(d=12 \mu \mathrm{m})$. The results of the two sets of measurements were in agreement. In Fig. 3, we show the temperature dependence of the birefringence in the pure LC and in one colloidal sample $\left(c_{\text {part }} \approx 0.05 \%\right)$. At $32^{\circ} \mathrm{C}$ the predicted $n_{a}^{C O L} / n_{a}^{L C}=1.2$ agrees with the experimental value $S_{C O L} / S_{L C}=1.2$. Thus, the birefringence increase in the ferroelectric colloid is consistent with the order parameter increase. We find that at $32^{\circ} \mathrm{C}, \varepsilon_{a}^{C O L} / \varepsilon_{a}^{L C}=1.54$. This result is close to, but not identical to $\left(n_{a}^{C O L}\right)^{2} /\left(n_{a}^{L C}\right)^{2} \approx S_{C O L}^{2} / S_{L C}^{2}=1.44$. We speculate that this discrepancy may be due to an extra contribution from the ferroelectric colloidal particles to the effective dielectric function of the colloid [14].

We speculate that the birefringence gain is a consequence only of the increase in the nematic ordering. If this is the case, then the ratio of the colloidal low frequency (i.e. $\nu \approx 1 \mathrm{kHz}$ ) dielectric constant to that in the pure LC should be the same as that at optical frequencies: $\varepsilon_{a}^{C O L} / \varepsilon_{a}^{L C}=\left(n_{a}^{C O L}\right)^{2} /\left(n_{a}^{L C}\right)^{2} \approx S_{C O L}^{2} / S_{L C}^{2}$. We used a Hewlett-Packard 4194A impedance analyzer to measure the LC cell capacitance. The cells were $15 \mu \mathrm{m}$ thick, and the dielectric anisotropy was determined by comparing results with planar and homeotropic orientation. In Fig. 4, we present results for the temperature dependence of the dielectric anisotropy $\varepsilon_{a}(T)$. 


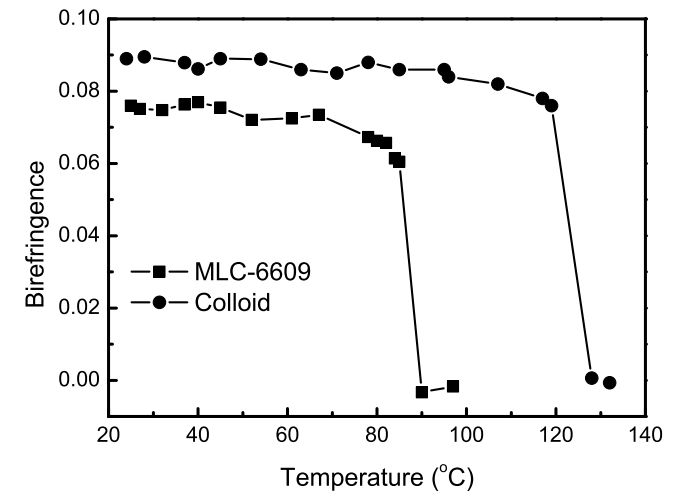

FIG. 3: Temperature dependence of the birefringence of MLC6609 and the ferroelectric colloid ( $\mathrm{c}_{\text {part }} \approx 0.05 \%$ ).

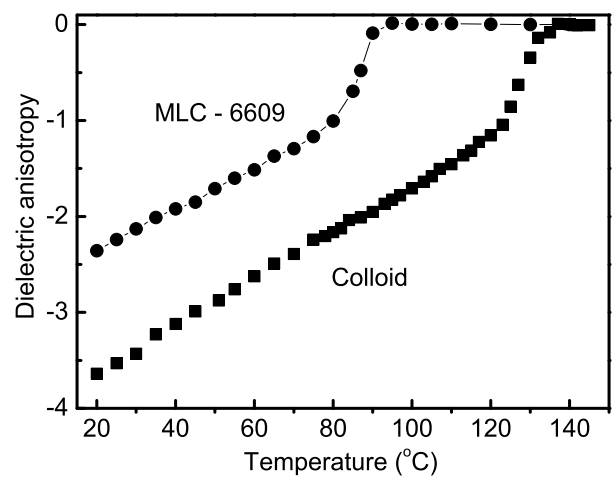

FIG. 4: Temperature dependences of the dielectric anisotropy of MLC-6609 and the ferroelectric colloid ( $\mathrm{c}_{\text {part }} \approx 0.2 \%$ ).

Smaller increase in the clearing point have been observed for other LC host systems (for example, Zli-2248, ZLI-4801 and 5CB), doped with both $\mathrm{Sn}_{2} \mathrm{P}_{2} \mathrm{~S}_{6}$ and $\mathrm{BaTiO}_{3}$ nanoparticles. The increases are always accompanied by increases in the dielectric anisotropy and birefringence.

All our experiments are consistent with an increase in the effective orientational interaction, $\mathcal{U}$. According to the Maier-Saupe theory [15], for our principal sample $\mathcal{U}_{C O L} / \mathcal{U}_{L C}=T_{N I}^{C O L} / T_{N I}^{L C} \approx 1.1$. In order to make further progress, we consider the additional terms in the free energy of the doped nematic LC resulting from the presence of the colloidal particles. We assume for simplicity that the ferroelectric particles are spherical in shape. A ferroelectric particle possesses a large permanent polarization, $\mathbf{P}$, which creates an electric field in its neighborhood [16]:

$$
\mathbf{E}_{\text {part }}=\frac{R^{3}}{3 \varepsilon_{0}}\left(3 \frac{(\mathbf{P} \cdot \mathbf{r}) \mathbf{r}}{r^{5}}-\frac{\mathbf{P}}{r^{3}}\right)
$$

where $R$ is the particle radius. This electric field induces a dipole moment in the host LC molecules, $\mu_{\text {ind }}=\varepsilon_{0} \beta$. $\mathbf{E}_{P} \sim 1 / r^{3}$, where $\beta$ is the tensor of molecular polarizability at low frequency. The interaction between the electric field $\mathbf{E}_{P}$ and the induced dipole $\mu_{\text {ind }}$ in LC molecules is given by $U_{L C-P} \sim \sum \varepsilon_{0} \beta_{i} \cdot \mathbf{E}_{P}\left(\mathbf{r}_{i}\right) \mathbf{E}_{P}\left(\mathbf{r}_{i}\right)$. The induced dipole moments in LC molecules also interact with each other. If $\varepsilon_{0} \beta_{i} \cdot \mathbf{E}_{P}\left(\mathbf{r}_{i}\right)=\mathbf{G}_{i}$ and $\mathbf{r}_{i}-\mathbf{r}_{j}=\Delta \mathbf{r}_{i, j}$, this additional intermolecular interaction is given by:

$U_{C O L}^{a d d} \sim \sum_{\substack{i, j, j \neq i, \\ \text { particles }}} \frac{\mathbf{G}_{i}}{4 \pi \varepsilon_{0}}\left[\frac{\mathbf{G}_{j}}{\left|\Delta \mathbf{r}_{i, j}\right|^{3}}-3 \frac{\left(\mathbf{G}_{j} \cdot \Delta \mathbf{r}_{i, j}\right) \Delta \mathbf{r}_{i, j}}{\left|\Delta \mathbf{r}_{i, j}\right|^{5}}\right]$

We now suppose the most important contributions come from nearest neighbor molecules. Averaging eq.(4) over LC molecule orientations yields:

$U_{a} \sim \frac{1}{2} N \sum_{\substack{i, \\ \text { particles }}} \frac{\left(\varepsilon_{0} \beta_{a} S_{C O L}\right)^{2}\left(\mathbf{E}_{P}\left(\mathbf{r}_{i}\right)\right)^{2}}{4 \pi \varepsilon_{0} L^{3}} \equiv-\frac{1}{2} e S_{C O L}^{2}$

where $N$ is the number of nearest neighbor LC molecules, $\beta_{a}$ is the anisotropy of LC molecule polarizability at low frequencies and $L$ is the nearest-neighbor distance. The key parameter $e$ is the proportionality coefficient characterizing the response of the intermolecular interaction to the field due to the ferroelectric particle.

In the Maier-Saupe molecular field theory, the intermolecular interaction energy takes the form $F_{1}=-\frac{1}{2} \mathcal{U} S^{2}$ [17]. The parameter $\mathcal{U}$ is related to the nematic-isotropic phase transition temperature by the ratio, $k_{B} T_{N I}=$ $0.22 \mathcal{U}$. This corresponds to intermolecular interaction per unit volume $\mathcal{U} N_{L C} \approx 4.7 \times 10^{7} \mathrm{~J} \mathrm{~m}^{-3}$, where $N_{L C}$ is the LC molecular concentration. Therefore, we derive a result for the change in the transition temperature due to a change in the molecular interaction parameter, $\Delta T_{N I} \approx$ $e \cdot T_{N I} \cdot\left(\mathcal{U} N_{L C}\right)^{-1}$. We now derive an explicit formula for the quantity $e$. In eq. (5) the main contribution to the sum comes from those molecules surrounding a given ferroelectric particle. We calculate the sum explicitly, concentrating on these LC molecules, giving rise to the following formula:

$$
e \approx \frac{1}{27} N \cdot N_{L C} \cdot N_{\text {part }} \frac{\beta_{a}^{2} P^{2}}{\varepsilon_{0}}\left(\frac{R}{L}\right)^{3}
$$

where $N_{\text {part }}$ is the volume concentration of particles. To make quantitative estimates, we take $P=0.26 \mathrm{C} \mathrm{m}^{-2}$, $N_{\text {part }}=7 \times 10^{19} \mathrm{~m}^{-3}, N_{L C}=2 \times 10^{27} \mathrm{~m}^{-3}, \quad N=6$, $R=50 \mathrm{~nm}$, and $L=0.5 \mathrm{~nm}$. Using $\beta_{a} N_{L C} \approx \varepsilon_{a}$ [13] 
and $\varepsilon_{a} \approx 1$, we find $e \approx 0.9 \times 10^{7} \mathrm{~J} \mathrm{~m}^{-3}$. The resulting shift $\Delta T_{N I} \approx 70^{\circ} \mathrm{C}$ is in qualitative agreement with our experimental observations. Our model does not consider other possible contributions to the intermolecular interaction. There may be image forces or quadrupole interactions that remain important even above the Curie temperature. These may be significant as a result of the high nanoparticle dielectric constant, causing $\Delta T_{N I}$ to be underestimated.

The observed effect requires $e$ as large as possible. From eq.(6) this is governed by the large ratio $(R / L)^{3} \approx 10^{6}$. For molecular, as opposed to colloidal, dopants this ratio would be of the order of unity, dramatically reducing the effect $e / a=\Delta T_{N I} \sim 10^{-4}{ }^{\circ} \mathrm{C}$. It is the supramolecular size of the impurity particles which causes the huge effect. In principle, this is testable by varying the particle size. The effect is proportional to the particle surface area, but should not grow without limit. Large particles generate orientational defects in their neighborhood, which destroy the colloidal homogeneity and lower orientational order. We estimate the critical particle size $R_{\max }=l \sim K / W$ ( $K$ is a Frank constant) [2]. For typical values $K=10^{-11} \mathrm{~N}$ and $W=$ $10^{-4} \mathrm{~J} \mathrm{~m}^{-2}, R_{\text {max }}$ is predicted as $100 \mathrm{~nm}$.

In sufficiently large colloidal particles, the ferroelectric material is expected to form a polydomain structure, which will further reduce the effective interaction below the ideal $R^{2}$ dependence. In small systems, the ferroelectric mean field will be insufficient to maintain a ferroelectric ground state. Ferroelectricity usually vanishes for particles of dimensions smaller than $\sim 10 \mathrm{~nm}$. We therefore speculate the optimal size of the ferroelectric particles in homogeneous LC colloids to be in the range (10-100) $\mathrm{nm}$.

In conclusion, our results clearly show the unique properties of ferroelectric nanoparticles/LC colloids. Since the particle size is much larger than the LC molecular dimension, the strong electric field from the particles influences a large number of neighboring LC molecules $\left(\sim 10^{3}\right)$. The huge ferroelectric dipole moment of the nanoparticles produces a powerful field inducing dipolar intermolecular interactions that compete with the spontaneous intermolecular interaction. The additional interaction then leads to a dramatic increase in the LC mean field interaction and thence to a giant increase in the clearing temperature.This also causes the homogeneous ferroelectric LC colloid to have a higher birefringence, dielectric anisotropy and order parameter than the pure LC material. These results are not only true in the model systems which we have investigated in detail, but are a general feature of colloidal suspensions made from ferroelectric colloidal particles dispersed in thermotropic liquid crystal matrices. As a result ferroelectric nanoparticles/LC colloids offer a simple and effective means to control the physical properties of liquid crystalline materials.

This work was supported by: NSF through Grant No. DMR-0508137; Hoseo University, through the academic research fund in 2004; the National Academy of Science of Ukraine (project "Composite liquid crystal and polymer materials for information technologies"); the Royal Society of London, through a joint Anglo-Ukrainian cooperation grant; and a NATO Collaborative Linkage Grant.

[1] F. Brochard and P-.G. de Gennes, J. Phys. (France) 31, 691 (1970).

[2] H. Stark, Phys. Rep. 351, 387 (2001).

[3] P. Poulin, H. Stark, T. C. Lubensky, and D. A. Weitz, Science 275, 1770 (1997).

[4] S. P. Meeker, W. C. K. Poon, J. Crain, and E. M. Terentjev, Phys. Rev. E 61, R6083 (2000).

[5] A. Glushchenko, H. Kresse, V. Reshetnyak, Y. Reznikov, and O. Yaroshchuk, Liq. Cryst. 23, 241 (1997).

[6] B. J. Liang and S. H. Chen, Phys. Rev. A 39, 1441 (1989).

[7] Y. Reznikov, O. Buchnev, O. Tereshchenko, V. Reshetnyak, A. Glushchenko, and J. West, Appl. Phys. Lett. 82, 1917 (2003).

[8] E. Ouskova, O. Buchnev, V. Reshetnyak, Y. Reznikov, and H. Kresse, Liq. Cryst. 30, 1235 (2003).

[9] Y.Reznikov, O.Buchnev, A.Glushchenko, V.Reshetnyak, O.Tereshchenko, and J.West, SPIE Proceedings 5741, 171 (2005)

[10] C.I. Cheon, L. Li, A. Glushchenko, J.L. West, Yu. Reznikov, J.S.Kim and D.H. Kim., SID'05 DIGEST 45.2, 1 (2005).

[11] D. E. Martire, G. A. Oweimreen, G. I. Ågren, S. G. Ryan, and H. T. Peterson, J. Chem. Phys. 64, 1456 (1976).

[12] B. Jaffe, W.R. Cook, Jr. and H. Jaffe, Piezoelectric Ceramics (Academic Press Limited, 1971).

[13] L.M. Blinov and V.G. Chigrinov., Electrooptic Effects in Liquid Crystal Materials (Springer, 1996).

[14] V.Yu. Reshetnyak, Mol. Cryst. Liq. Cryst. 421, 219 (2004).

[15] P-.G. de Gennes and J. Prost, The Physics of Liquid Crystals (Oxford University Press, New York, 1994).

[16] L. D. Landau, E. M. Lifshitz, and L.P. Pitaevskii, Electrodynamics of continuous media, Chap. II. (Pergamon Press, New York, 1984).

[17] M.J. Stephen and J.P. Straley, Rev. Mod. Phys., 46, 617 (1974). 